\title{
Comportamiento ecológico de los gestores de campos de golf en Andalucía (España)
}

\author{
Jesus Manuel López-Bonilla, Luis Miguel López-Bonilla, \\ Borja Sanz-Altamira, Maria del Carmen Reyes-Rodríguez
}

\section{RESUMEN}

La práctica del golf tiene un gran potencial turístico en países como España o en regiones como Andalucía. Pero es necesario cuidar de su desarrollo para que se haga de una manera racional y equilibrada. Recientemente, la Comunidad Autónoma de Andalucía ha aprobado una nueva normativa que regula las condiciones de implantación y funcionamiento de los campos de golf andaluces. Este trabajo presenta los resultados de un estudio realizado sobre la sostenibilidad de los campos de golf en Andalucía desde la perspectiva empresarial. Entre los principales resultados, se puede destacar que los directivos de campos de golf se sienten motivados en su comportamiento ecológico fundamentalmente por la necesidad de satisfacer aspectos empresariales (imagen y responsabilidad social), quedando en un segundo plano otros factores propios de su entorno, como las demandas de los jugadores, la influencia de la legislación y la de los grupos ecologistas.

PALABRAS-CLAVE: Gestión Medioambiental; Golf; Turismo.

\section{Behavior green of the managers of golf courses in Andalusia (Spain)}

\section{ABSTRACT}

Golf offers great potential for tourism in countries such as Spain or in regions like Andalusia (an Autonomous Community of Spain). But we must ensure that its development is done in a rational and balanced manner. Recently, the local government of Andalusia has issued new regulations governing the implementation and operation of golf courses in the region. This paper presents the results of a study on the sustainability of golf courses in Andalusia from a business perspective. One of the key results is that golf course managers are motivated in their environmental performance mainly by the need to meet business goals: image and social responsibility; being in the background other factors related with their environment, such as the demands of the players, the influence of legislation and environmental groups.

KEYWORDS: Environmental Management; Golf; Tourism. 


\section{Introducción}

La práctica del golf tiene una importancia creciente en el desarrollo turístico mundial. El turista que incluye la práctica del golf en sus viajes suele realizar un elevado gasto medio durante sus días de estancia, por lo que se convierte en un tipo de demanda muy deseable para las zonas turísticas. No obstante, es necesario que la oferta de golf se haga de manera racional, respetando el entorno donde se ubica, con idea de mantener su equilibrio social, económico y medioambiental en el futuro.

Como ya indicaba Turespaña (2004), en su estudio sobre el mercado europeo de turismo de golf, España goza de una clara y destacada posición de liderazgo con una cuota de mercado cercana al $35 \%$. En este contexto, Andalucía es la Comunidad Autónoma española que dispone actualmente de un mayor número de campos de golf y, por tanto, es el principal destino de turismo de golf de España. No obstante, esta región ha experimentado un gran crecimiento del turismo de golf en los últimos años, sobre todo, en el litoral. Por ejemplo, la Costa del Sol suele ser también reconocida, en términos coloquiales, como la Costa del Golf.

El gobierno andaluz aboga por el desarrollo de un producto turístico, a través de los campos de golf, que diversifique la oferta, aumente los ingresos por turista y reduzca la estacionalidad. Pero debe tener en consideración su estrecho vínculo con el entorno medioambiental. En este sentido, la Comunidad Autónoma de Andalucía ha aprobado recientemente el Decreto 43/2008 con idea de regular las condiciones de implantación y funcionamiento de campos de golf en Andalucía. Como se describe en su preámbulo, este Decreto no sólo responde a la importancia deportiva, turística o económica del fenómeno, sino que también atiende a sus dimensiones medioambientales o urbanísticas que se proponen encauzar de forma positiva, compatibilizando la promoción del golf como nuevo eje de desarrollo deportivo y turístico con la preservación del patrimonio natural, la reducción de impactos territoriales o medioambientales y, cuando lo posibiliten los ámbitos de implantación, con la mejora y regeneración de los entornos naturales. Con ello, se pretende fomentar la mejora y el respeto al medio natural, la restauración y protección del paisaje, el uso de suelos o zonas degradadas, la utilización de sistemas de gestión medioambiental eficaces, el uso de energías renovables y la minimización de la contaminación y de las emisiones, compaginándose todo ello con un escrupuloso respeto a las normas y principios de protección del suelo, de ordenación territorial y urbanística y de la salud pública.

El presente trabajo forma parte de un proyecto de investigación más amplio que se ocupa de estudiar la sostenibilidad del turismo de golf en Andalucía (España). En este artículo presentamos los resultados obtenidos del estudio realizado a los gestores de campos de golf en Andalucía. Tratamos de comprender la visión empresarial de la materia prima del turismo de golf, como son los propios campos de golf, en torno a su orientación hacia los retos que supone la implicación medioambiental en el desarrollo de su actividad. 


\section{Impacto medioambiental del turismo de golf}

Se sabe que el turismo de golf genera riqueza pero también es frecuente la controversia de los campos de golf debido a que se cuestiona su estrecha relación con el medio ambiente y, en consecuencia, su posible efecto negativo sobre el mismo. No obstante, los impactos negativos provocados por los campos de golf en la mayor parte de los casos son originados por los errores que se han cometido en el inicio del proyecto, o bien, por los errores consecuentes que ocasiona de su mantenimiento. La práctica del golf es lo suficientemente flexible como para no presuponer una agresión al entorno, sino más bien todo lo contrario. Como indica Espejo (2004, p. 79), "un campo de golf adecuadamente planificado, diseñado, construido y mantenido, además de ser respetuoso con el medio natural, contribuye a realzar su belleza".

Se entiende por impacto ambiental la alteración que la ejecución de un proyecto introduce en el medio, expresada por la diferencia entre la evolución de éste sin y con proyecto (BORREGO, 2002).

Las preocupaciones medioambientales en torno a los campos de golf giran fundamentalmente en torno a los siguientes aspectos (PANIZA, 2005):

- Cambios en los usos de las tierras y movimientos en el terreno que pueden alterar hábitats de la fauna silvestre, humedales, corrientes y estanques.

- Uso de fertilizantes químicos y pesticidas que pueden contaminar la tierra, el agua -superficial y subterránea- y dañar a plantas y animales autóctonos.

- Consumo elevados de agua para abastecimiento del campo de golf.

Priestley y Sabí (1993), en su investigación sobre el medio ambiente y el golf en Cataluña, distinguen cuatro manifestaciones de impacto ambiental generado por la implantación de los campos de golf en ese ámbito territorial: (1) el cambio cualitativo del paisaje; (2) la problemática del valor ecológico; (3) el efecto de los modelos de construcción de los campos de golf; y (4) el suministro de agua.

Videira et al. (2006), en su estudio sobre el golf en el Algarve (Portugal), consideran que para que la industria del golf sea sostenible debe cumplir con los requisitos que se mencionan a continuación.

- Que sea competitiva, por su capacidad de mantenerse en el mercado, generando beneficios a medio plazo, ofreciendo servicios y productos de elevada calidad.

- Que sea ambientalmente responsable, ya que es consumidora de recursos naturales, fundamentalmente suelo y agua, además de servicios intangibles como paisaje, biodiversidad o clima. Una protección de estos recursos es vital para que el golf presente ventajas competitivas insustituibles.

- Que sea generadora de impactos sociales y económicos positivos, debido a 
que el desarrollo equilibrado del golf junto con los sectores turísticos y de la construcción puede contribuir a generar impactos significativos a la economía.

- Que esté integrada en el desarrollo regional, ya que el golf es una actividad consumidora extensiva de suelo, por lo que la localización de los campos de golf condiciona y está condicionada por la ordenación del territorio, por sus usos y aptitudes.

Los efectos que el turismo de golf puede ocasionar sobre el medio ambiente (consumo de agua, empleo de plaguicidas, etc.) está motivando una mayor atención tanto por parte de la Administración Pública como por parte de las empresas (Espejo, 2004). Así, por un lado, cada vez está más extendida la obligatoriedad de llevar a cabo un estudio de impacto ambiental de los nuevos proyectos de golf, y por otro, los gestores de los campos de golf comienzan a desarrollar nuevas estrategias de sostenibilidad, aceptando la idea de que un conocimiento adecuado de la incidencia ambiental de esta actividad es fundamental para una correcta gestión de los mismos: la variable medioambiental empieza a ser considerada a nivel estratégico por parte de la dirección de estas organizaciones (VARGAS; VACA; GARCÍA, 2004b).

El Sistema de Gestión Medioambiental (SGMA) es una herramienta que cada vez cobra más fuerza en la gestión ambiental ya que puede prevenir, reducir, e incluso eliminar los impactos medioambientales negativos que puedan ser ocasionados por la actividad que genera la construcción y el mantenimiento de los campos de golf. La instalación de este sistema puede realizarse según el criterio de la empresa, pero lo ideal es acogerse a sistemas normalizados ya que proporciona transparencia a la gestión y permite la utilización de marcas de gran interés en la promoción comercial. Todos los sistemas se basan en una mejora continua y progresiva del medio ambiente, así como en una transparencia de cara a la opinión pública, las instituciones y los clientes. Los sistemas más reconocidos son el EMAS (Sistema Comunitario de Ecogestión y Ecoauditoría) y la norma ISO-14001. Esta norma es un estándar de calidad reconocido internacionalmente que establece los requisitos mínimos que debe reunir un Sistema de Gestión Ambiental. Los sistemas mencionados aportan una serie de beneficios a las organizaciones, como pueden ser las mejoras de imagen y comunicación, la reducción de costes o el aumento de la competitividad.

La implantación de un Sistema de Gestión Medioambiental no sería viable sin la preparación técnica de los empleados del propio campo de golf. No sólo es suficiente una declaración de intenciones por parte de los gestores, sino que se hace necesario que se realice una apuesta en firme por la formación y la preparación del personal que va a formar parte de ese plan de gestión medioambiental (PANIZA, 2005).

Los campos de golf tienen que ser tratados como casos únicos, dado que las condiciones naturales son diferentes de un lugar a otro, aunque haya una serie de aspectos ambientales que sea común para todos los campos al plantearse su planifica- 
ción, diseño, construcción y gestión de los campos de golf, con el fin de evitar, en la medida de lo posible, cualquier impacto negativo tanto en el paisaje como en los recursos naturales. Por ello, como indica Escorza (2001), es muy importante incluir, junto al proyecto de realización del campo de golf, un informe o estudio de impacto ambiental (en función de las características y dimensiones del campo) que asegure tanto la viabilidad del citado proyecto como las garantías de que el mismo no va a incidir de forma negativa en el entorno en el que se pretende ubicar.

Finalmente, si la sociedad demanda este tipo de actividad recreativa, debe tenerse en cuenta y poderse satisfacer de la mejor manera posible. Por ello, un desarroIlo ambientalmente sostenible de los campos de golf debe tender a la búsqueda y ubicación en lugares con aptitudes para ello, de manera que se pueda cubrir la demanda social con el menor grado de impacto para el entorno. Pero también hay que considerar en gran medida las propias actitudes de los gestores de campos de golf para potenciar las prácticas medioambientales.

\section{Objetivos y metodología}

El presente estudio se ha basado en las actitudes y comportamientos medioambientales de los gestores de campos de golf en Andalucía. Para ello, se ha planteado una serie de cuestiones tanto generales como personales del encuestado. Las opciones de respuestas se han construido en función de una escala de Likert de cinco alternativas que van desde muy de acuerdo (5) hasta muy en desacuerdo (1). Estas cuestiones se han elaborado a partir de la revisión de la literatura existente en la materia. Uno de los bloques de preguntas está relacionado con la puesta en funcionamiento de un sistema de gestión medioambiental, que se ha basado en las normas ISO-14001.

Por otro lado, la muestra se ha compuesto de 75 directivos de campos de golf andaluces. El método de contacto ha sido a través de encuesta telefónica y se ha realizado durante el mes de abril de 2009. Se ha contado con el censo de 98 campos de golf que tiene Andalucía, lo que indica que ha habido una elevada participación de directivos que ha realizado la encuesta. En concreto, ha alcanzado al $76,5 \%$ del total de la población estudiada.

\section{Análisis de datos}

Como ya se ha comentado anteriormente, se realizaron en total 75 encuestas a los directivos de los campos de golf en Andalucía. A continuación se exponen los resultados, donde se muestra y se comenta una serie de gráficos obtenidos a partir de los datos recogidos en dichos cuestionarios. 


\section{Preocupación medioambiental de los gestores}

Los gestores consideran que las causas que más influyen en su preocupación medioambiental son, en primer lugar, el deseo de transmitir una imagen favorable de la empresa en la que trabajan y, en segundo lugar, la responsabilidad social de la organización. Tanto uno como otro motivo están muy relacionados con la imagen corporativa que a los negocios de este sector empresarial les interesa transmitir a la sociedad.

Las causas relativas a la presión de los jugadores (tercera en importancia), la presión legal (cuarta en importancia) y la presión ecologista, tienen un menor peso, destacando la presión de las organizaciones ecologistas como la menos influyente en la preocupación medioambiental de los gestores de los campos de golf.

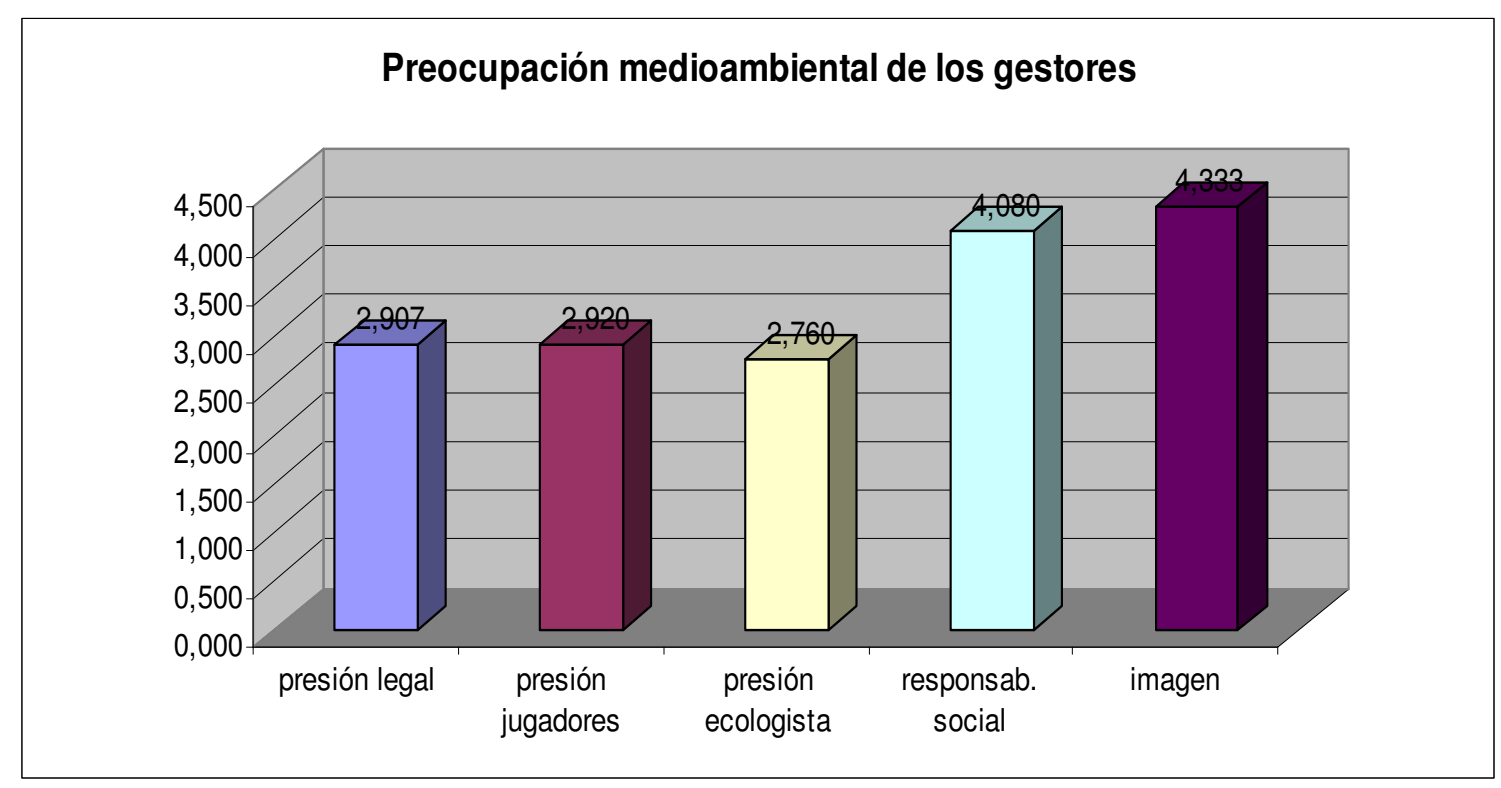

Figura 1: Causas de la preocupación medioambiental de los gestores

\section{Percepción de los directivos sobre los factores que demandan los jugadores de golf}

Los gestores piensan que el factor más importante para el jugador es el cuidado del campo de golf. Sitúan en segundo lugar el precio. En tercer lugar, la cercanía al lugar de residencia y, en cuarta posición, el diseño del campo. Distanciados considerablemente de estos cuatro factores aparecen las limitaciones relativas a la posible masificación del campo (quinto factor en importancia), la información sobre la naturaleza del área donde se asienta (sexto lugar) y, por último, la utilización correcta de los recursos naturales.

Los resultados muestran que los directivos tienen la percepción de que los ju- 
gadores no demandan características que podríamos considerar como más vinculadas al medioambiente, puntuando estos factores por debajo de la media del conjunto estudiado. Mientras que los aspectos relacionados con la conveniencia particular (cuidado, precio y cercanía) son los más relevantes. Estos resultados son coherentes con los obtenidos en la preocupación medioambiental de los gestores, donde la presión de los jugadores era considerada de poca importancia.

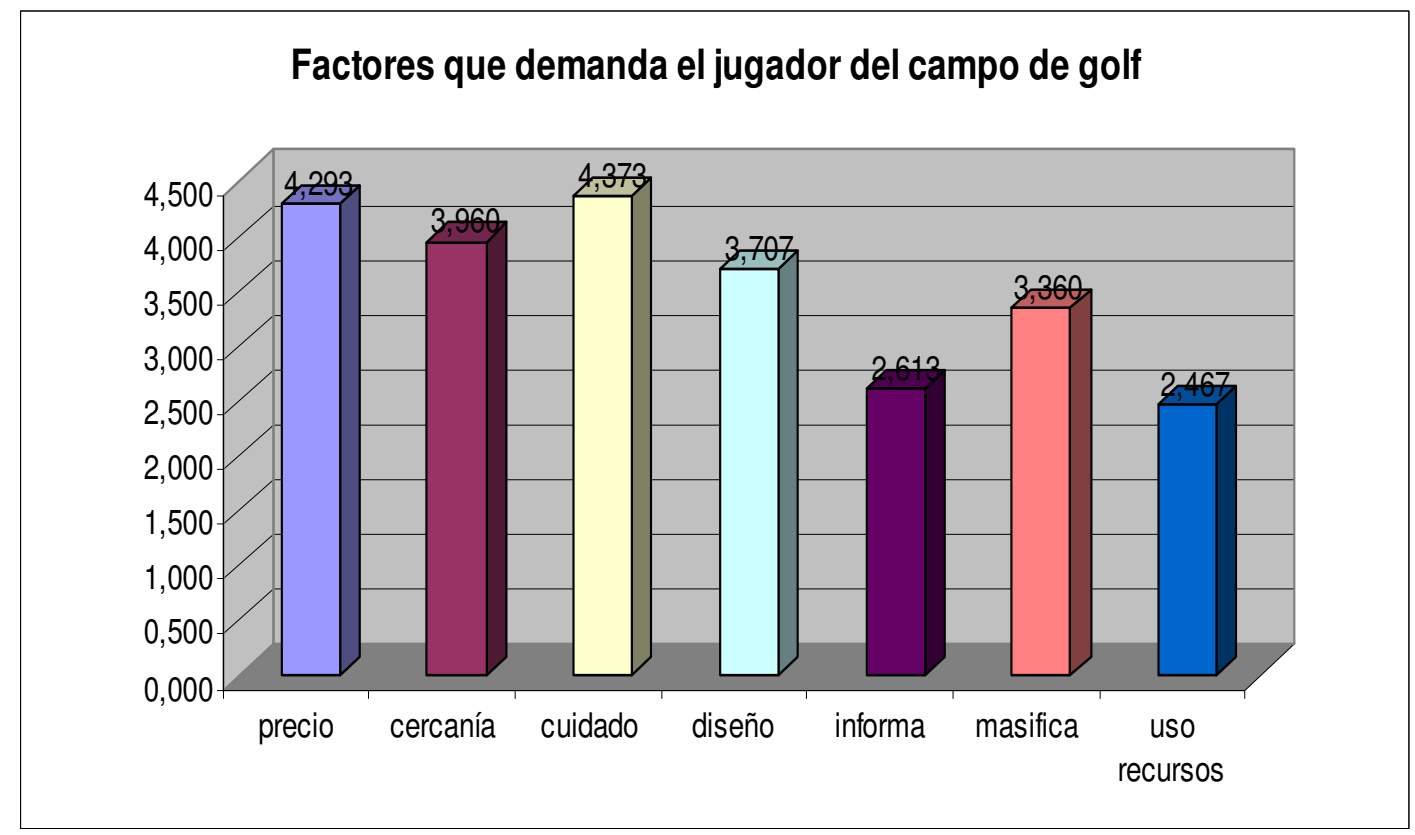

Figura 2: Factores generales que demandan los jugadores de golf según los gestores

\section{Actitudes medioambientales: inconvenientes de adoptar prácticas medioam- bientales según los gestores}

Con esta pregunta se pretende conocer a quiénes pueden perjudicar las prácticas que intentan conservar el medio ambiente. Para los gestores de campos de golf los principales perjudicados son los países del tercer mundo. En segundo lugar sitúan a la gestión de los campos de golf, en tercer lugar, la vida cotidiana de las personas y en cuarto lugar las economías de los países desarrollados. Por tanto, la opinión mayoritaria es que las economías de los países más pobres serían las más afectadas y las de los más ricos las menos.

Por otro lado, los directivos opinan que la gestión de los campos de golf estaría más afectada que las vidas cotidianas de las personas. No obstante, ninguna de las puntuaciones tienen valores altos, por lo que, en general, se puede decir que parecen opinar que la adopción de prácticas compatibles con el medio ambiente no tiene una repercusión muy negativa. 


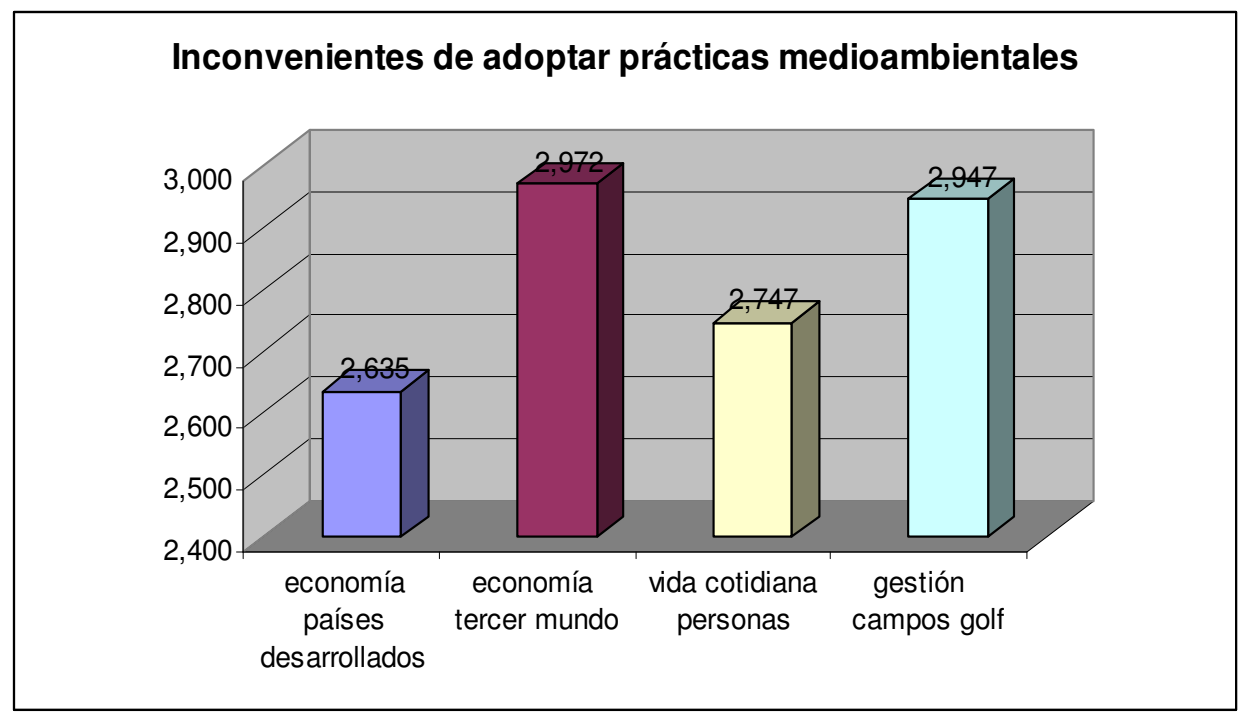

Figura 3: Inconvenientes de las prácticas medioambientales según los gestores

\section{Actitudes medioambientales: causas de los problemas medioambientales según los gestores}

Se intenta saber a qué factores se les atribuye la aparición de problemas en el medio ambiente. La causa principal es atribuida a la industria, en segundo lugar a las actividades de las personas, en tercero a la política del gobierno, siendo la menos puntuada el turismo. Así, las tres primeras tienen una puntuación alta, sobre todo, la primera de ellas. Mientras que la repercusión de las actividades turísticas es considerada como una causa menos relevante.

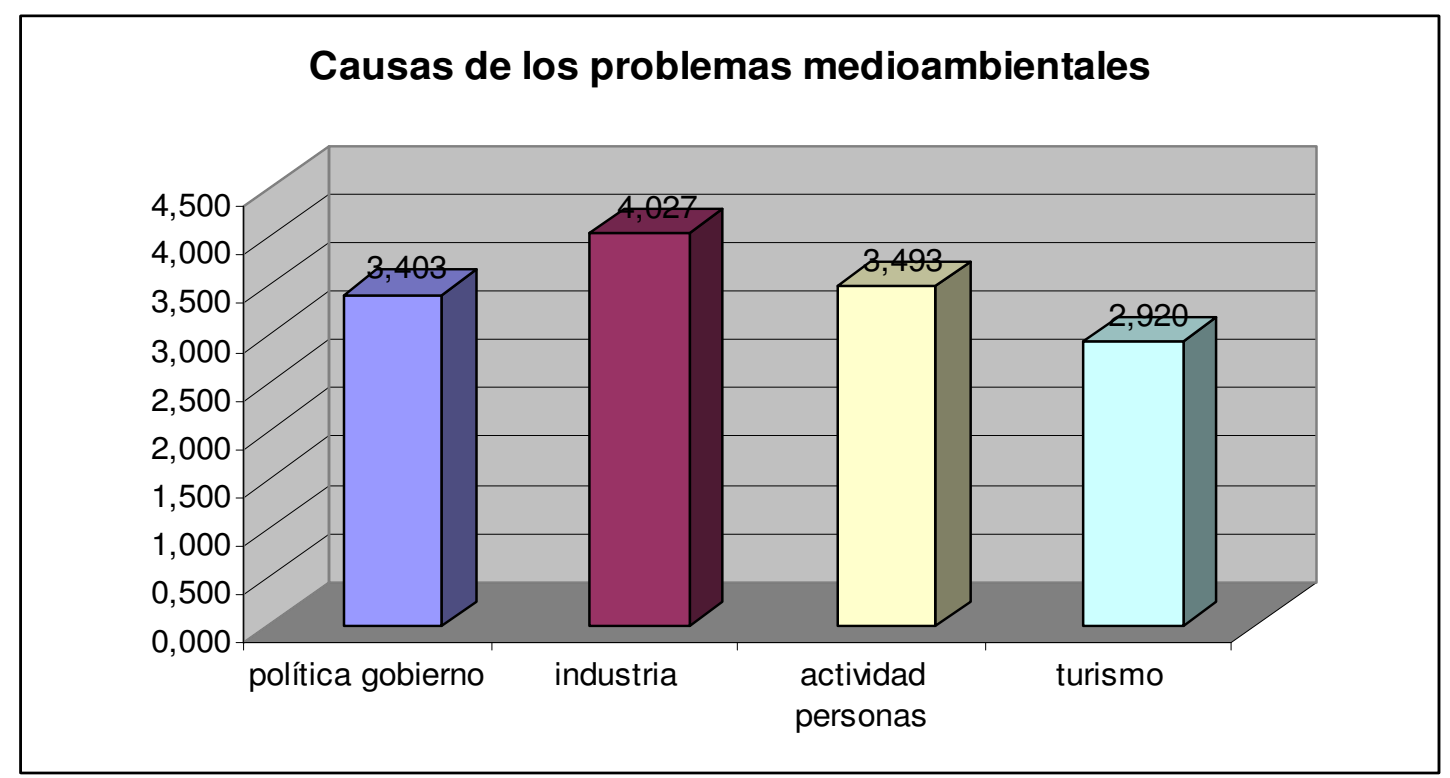

Figura 4: Causas de los problemas medioambientales según los gestores 


\section{Creencias medioambientales de los gestores}

Se pretenden conocer algunas opiniones relacionadas con la problemática medioambiental. Todas reciben una puntuación por encima de la media, esto es, superior a tres puntos. La más altamente puntuada es la opinión sobre la limitación de los recursos naturales, en segundo lugar el sometimiento de todos a las leyes de la naturaleza. La fragilidad del equilibrio medioambiental recibe la tercera de las puntuaciones más altas. La opinión de que estamos abusando gravemente del medio ambiente puntúa en cuarto lugar respecto a las demás, pero también tiene un valor alto. La opinión más catastrofista sobre el medio ambiente es la que recibe menor puntuación, pero, aún así, está por encima de la puntuación media (es decir, mayor de tres). En función de estas respuestas se puede considerar que, en general, los gestores manifiestan una alta concienciación sobre la limitación de recursos naturales y las consecuencias negativas aparejadas a su abuso.

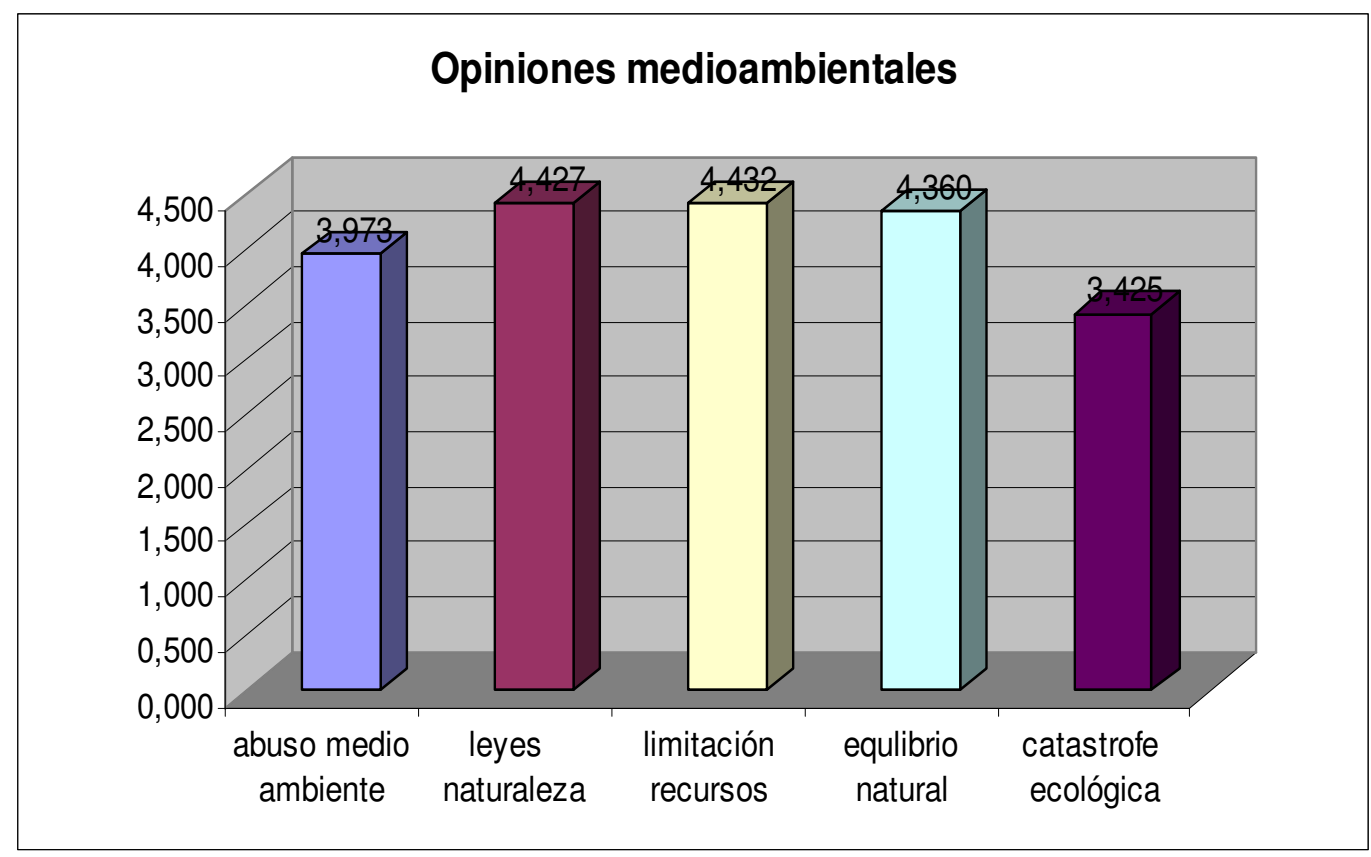

Figura 5: Opiniones medioambientales de los gestores

\section{Sistema de gestión medioambiental (sgma) de los campos de golf}

Estudiamos el cumplimiento de la norma ISO 14001 por las empresas del sector. El sistema se divide en cinco grandes apartados: política, planificación, implementación, verificación y revisión por parte de la dirección. Trabajando con las valoraciones promedio de cada uno de estos apartados estudiaremos cuáles son los que se aproximan más a los requisitos de las normas de los SGMA y cuáles son los que más se distancian. Los que poseen un mayor grado de aproximación a los estándares son la implementación, en primer lugar, y la planificación, en segundo lugar. La revisión por 
parte de la dirección tiene una distancia algo mayor, siendo el apartado de verificación y de política los más alejados. No obstante, todos los apartados tienen un grado medio y alto de aproximación a la norma.

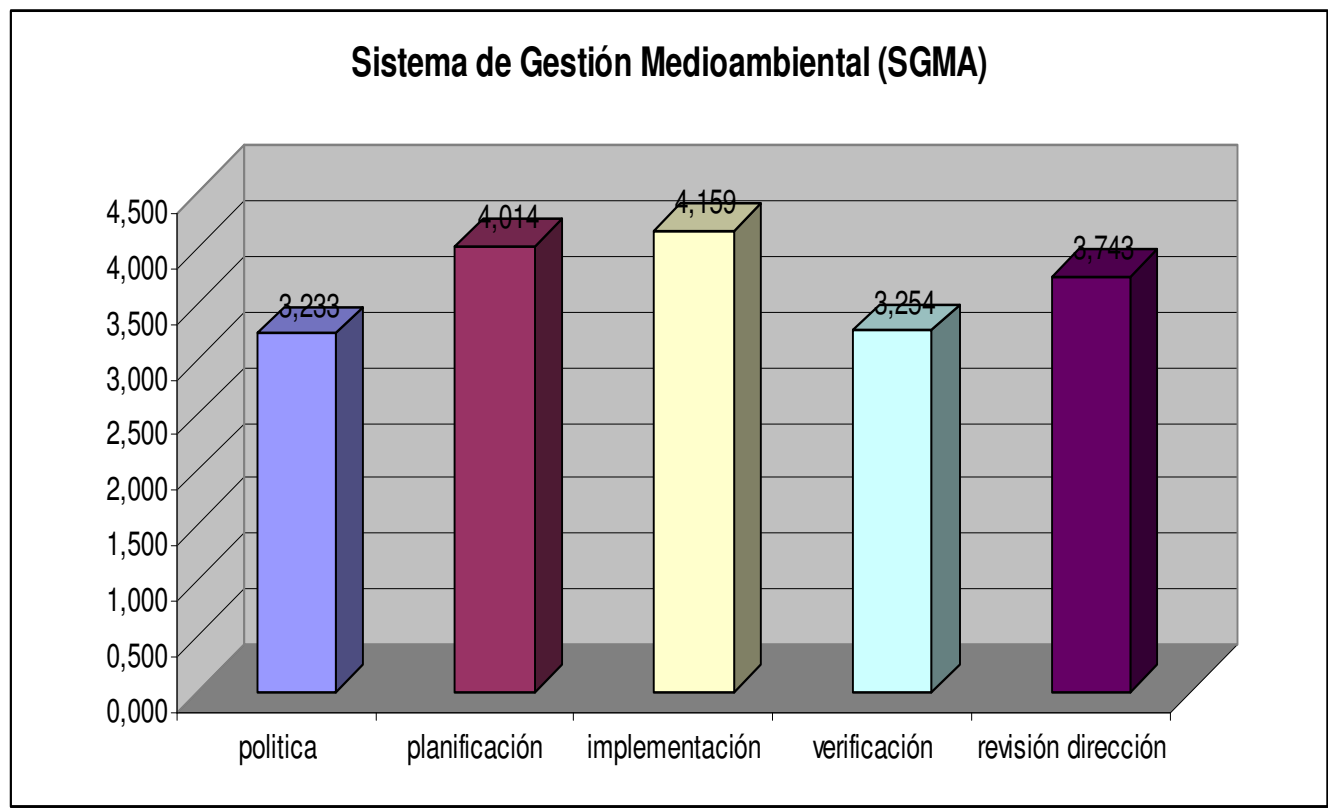

Figura 6: Sistema de Gestión Medioambiental (SGMA) de los campos de golf

Si analizamos con más detalle cada uno de estos cinco grandes bloques observamos que, dentro de la planificación, la verificación de las normativas legales posee un grado más óptimo que los objetivos y metas.

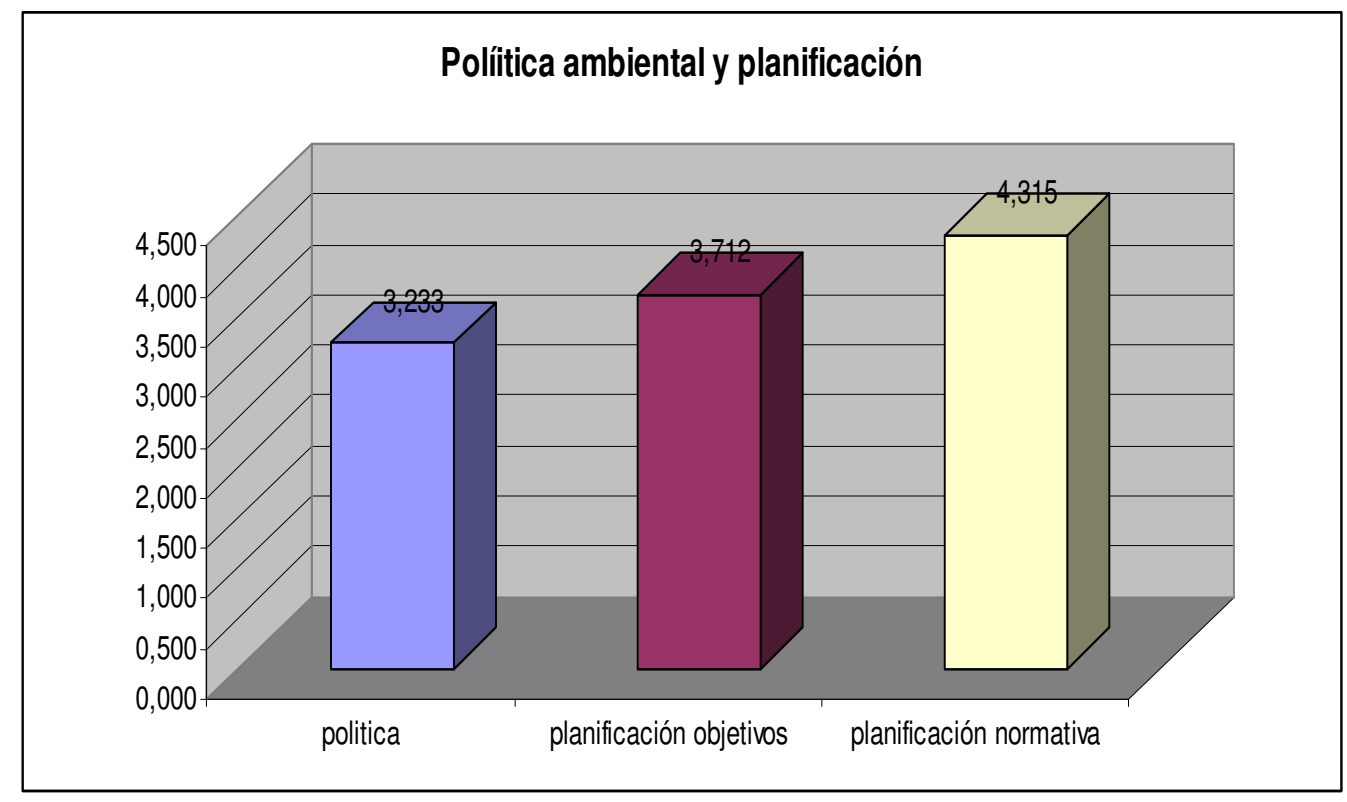

Figura 7: SGMA de los campos de golf: política ambiental y planificación 
En el apartado de implementación las mayores carencias se dan en la comunicación, en primer lugar, y la implementación de medidas de emergencia, en segundo lugar. La implementación de funciones y la concienciación de los empleados tienen un grado altísimo de aproximación a la norma.

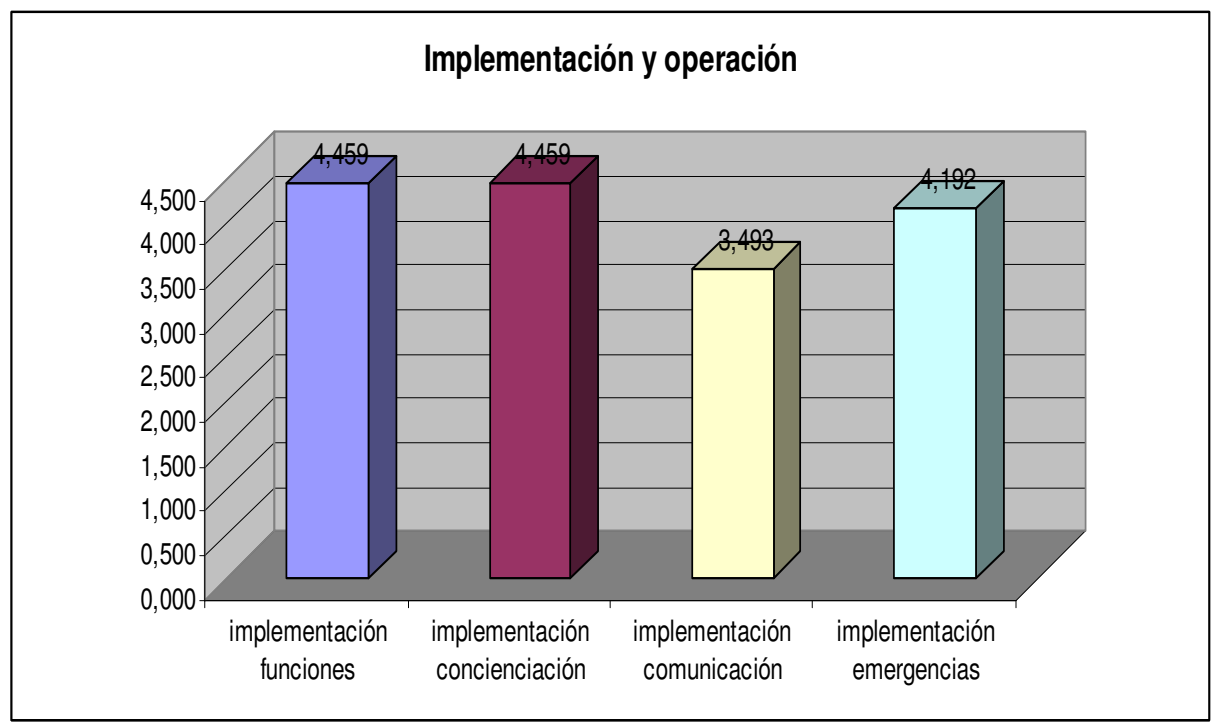

Figura 8: SGMA de los campos de golf: implementación y operación

Dentro del bloque de verificación, las carencias más importantes están en la realización de auditorías periódicas, estando mejor posicionadas la definición de responsabilidades y las competencias para acometer acciones correctoras, en primer lugar, y contar con un programa de control para verificar si se satisfacen los requisitos medioambientales, en segundo lugar.

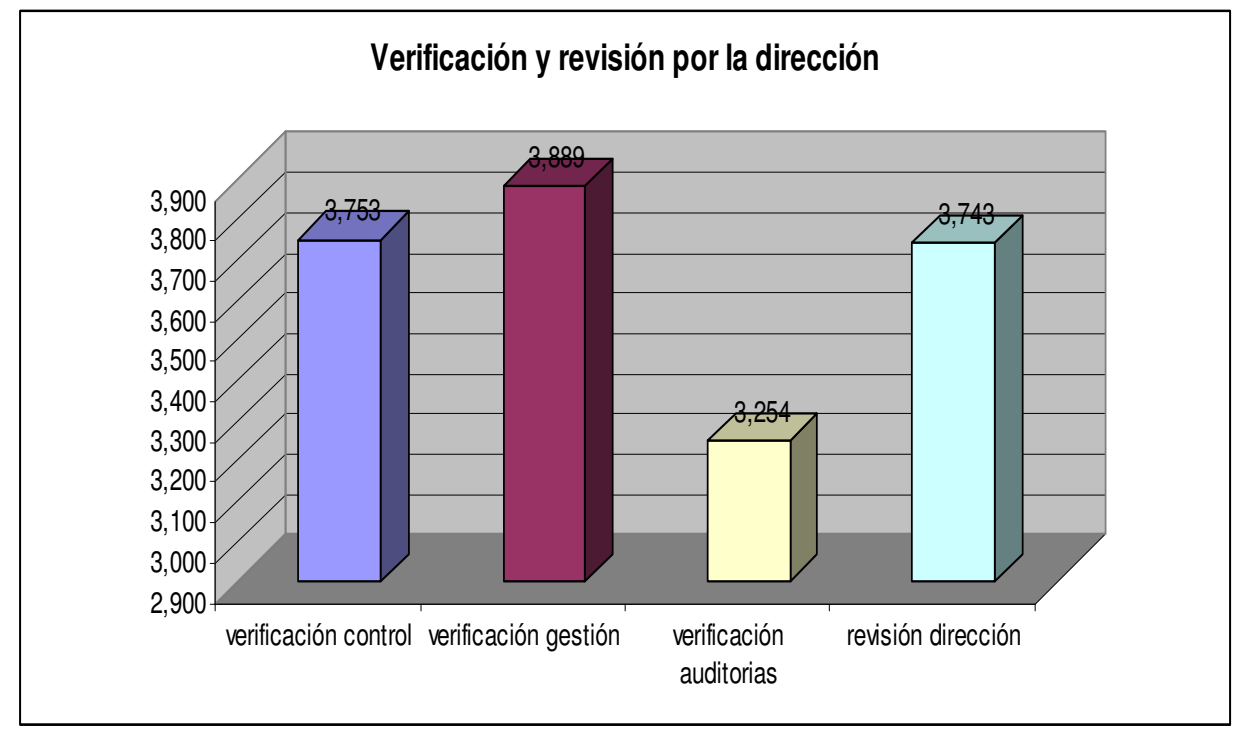

Figura 9: SGMA de los campos de golf: verificación y revisión por la dirección 


\section{Conclusiones}

A continuación se indican las principales conclusiones que se han extraído de los resultados descritos anteriormente. Los directivos se sienten motivados fundamentalmente por la necesidad de satisfacer aspectos empresariales (imagen y responsabilidad social), permaneciendo en un segundo plano otros factores propios de su entorno, como las demandas de los jugadores, la influencia de la legislación y la de los grupos ecologistas. Esto puede llevarnos a pensar que los gestores de los campos de golf actualmente se están anticipando a los cambios provenientes de su entorno, con una predisposición favorable a la hora de plantear acciones que eviten el deterioro del entorno natural, actuando de forma proactiva, esto es, anticipándose a los problemas, y no reactiva, es decir, obligados a reaccionar ante los mismos.

El gestor no percibe que la preocupación por el medio ambiente sea tan importante para el jugador de golf como pueden ser otros factores más vinculados al uso particular del servicio ofrecido.

Los gestores opinan que la adopción de prácticas compatibles con el medio ambiente no tiene una repercusión muy negativa para la economía de los países desarrollados y en vías de desarrollo, ni tampoco para la gestión de los campos de golf y la vida cotidiana de las personas. Aunque entre estos cuatro factores, consideran que tienen una mayor incidencia sobre los países menos desarrollados y en la gestión de los campos de golf.

Los gestores manifiestan una alta concienciación sobre la limitación de recursos naturales y las consecuencias negativas que conlleva su abuso. Opinan que la causa principal de los problemas medioambientales es el sector industrial, mientras que el turístico tiene una escasa repercusión.

Mediante el estudio del cumplimiento de la norma ISO 14001, nos hemos aproximado a la observación de la aplicación de un Sistema de Gestión Medioambiental (SGMA) por parte de las empresas de golf, concluyendo que los cinco grandes apartados en los que se estructura dicha norma son alcanzados en un grado medio 0 alto.

\section{Bibliografía}

BORREGO DOMÍNGUEZ, S. Campos de golf y turismo. Estudio de Málaga y su provincia. 2002. Tesis doctoral - Universidad de Málaga, Málaga, 2002.

CONSEJERÍA DE MEDIO AMBIENTE. Guía de Gestión Medioambiental de los Campos de Golf: Sistema de Gestión Medioambiental. Sevilla: Conserjería de Medioambiente de la Junta de Andalucía, 1996.

CONSEJERÍA DE TURISMO, COMERCIO Y DEPORTE. La demanda del turismo de golf en Andalucía. Sevilla: Junta de Andalucía, 2005. 
López-Bonilla, J.M.; López-Bonilla, L.M.; Sanz-Altamira, B.; Reyes-Rodriguez, M.C.

CONSEJERÍA DE TURISMO, COMERCIO Y DEPORTE. Plan General de Turismo Sostenible. Sevilla: Junta de Andalucía, 2007.

CONSEJERÍA DE TURISMO, COMERCIO Y DEPORTE. Balance del Año Turístico de Andalucía 2007. Sevilla: Junta de Andalucía, 2008.

CONSEJERÍA DE LA PRESIDENCIA. Decreto 43/2008, de 12 de febrero, regulador de las condiciones de implantación y funcionamiento de los campos de golf en Andalucía. BOJA, no 41, pp. 9-16, 2008.

ESCORZA DOBLAS, F. El turismo de golf en la Costa del Sol: análisis geográfico. Málaga: Diputación provincial del Málaga, 2001.

ESPEJO MARÍN, C. Campos de golf y medio ambiente: una interacción necesaria. Cuadernos de Turismo, 14, pp. 67-111, 2004.

PANIZA PRADOS, J.L. La Percepción Social del Golf en Andalucía, Sevilla: Consejería de Turismo, Comercio y Deporte de la Junta de Andalucía, 2005.

PRIESTLEY, G. K.; SABÍ BONASTRE, J. El medio ambiente y el golf en Cataluña: problemas y perspectivas, Documents d’ Analisi Geográfica, № 23, pp 45-74, 1993.

TURESPAÑA. Estudio sobre la demanda extranjera de golf: la promoción y comercialización del turismo de golf en España. Madrid: Publicaciones Profesionales de Turespaña, 2004.

VARGAS SÁNCHEZ, A.; VACA ACOSTA, R.M.; GARCÍA DE SOTO CAMACHO, E. Empresa turística y gestión medioambiental: una aplicación a los campos de golf de la provincia de Huelva, Estudios Turísticos, № 160, pp. 103-126, 2004a.

VARGAS SÁNCHEZ, A.; VACA ACOSTA, R.M.; GARCÍA DE SOTO CAMACHO, E. Turismo y gestión medioambiental. Diagnóstico de los campos de golf de la provincia de Huelva, Anais... VI Seminario Hispano-Luso de Economía Empresarial, Universidad de Huelva, Huelva, 2004b.

VIDEIRA, N.; CORREIA, I.; ALVES, I.; RAMIRES, C.; SUBTIL, R.; MARTINS, V. Environmental and economic tools to support sustainable golf tourism: The Algarve experience, Portugal, Tourism and Hospitality Research, 6(3), pp. 204-217, 2006.

\section{Agradecimientos}

Los autores de este artículo desean expresar su agradecimiento a la Consejería de Turismo, Comercio y Deporte de la Junta de Andalucía, que ha financiado la realización del proyecto de investigación en el que se enmarca este trabajo.

Por último, indicar que el origen de este trabajo proviene de la ponencia presentada en el XI Seminário Luso-Espanhol de Economia Empresarial (Faro, Portugal, 2009). 
Jesus Manuel López-Bonilla: Universidad de Sevilla.

Email: lopezbon@us.es

Link para o currículo: http://investigacion.us.es/sisius/sis showpub.php?idpers=5211

Luis Miguel López-Bonilla: Universidad de Sevilla.

Email: luismi@us.es

Link para o currículo: http://investigacion.us.es/sisius/sis showpub.php?idpers=2491

Borja Sanz-Altamira: Universidad de Sevilla.

Email: borja@us.es

Link para o currículo: http://investigacion.us.es/sisius/sis showpub.php?idpers=4934

Maria del Carmen Reyes-Rodríguez: Universidad de Sevilla.

Email: mcreyes@us.es

Link para o currículo: http://investigacion.us.es/sisius/sis showpub.php?idpers=4931

Data de submissão: 14 de juho de 2010.

Data do aceite: 17 de agosto de 2010 . 